\title{
Efeitos da vibração mecânica nas junções neuromusculares e tipo de fibra do músculo sóleo de ratas wistar ooforectomizadas*
}

\section{Effects of Mechanical Vibration in Neuromuscular Junctions and Fiber Type of the Soleus Muscle of Oophorectomized Wistar Rats}

\author{
Ana Luiza Peretti ${ }^{1}$ Camila Mayumi Martin Kakihata ${ }^{1}$ Maria Luiza Serradourada Wutzke ${ }^{1}$ \\ Márcia Miranda Torrejais ${ }^{1}$ Lucinéia de Fátima Chasko Ribeiro ${ }^{1}$ Gladson Ricardo Flor Bertolini ${ }^{10}$ \\ 1 Universidade Estadual do Oeste do Paraná - Campus Cascavel, \\ Cascavel, PR, Brasil \\ Rev Bras Ortop 2019;54:572-578. \\ Endereço para correspondência Gladson Ricardo Flor Bertolini, PhD, \\ Rua Universitária, Jardim Universitário Cascavel, PR, 85819110, Brasil. \\ (e-mail: gladson_ricardo@yahoo.com.br).
}

\section{Resumo \\ Palavras-chave \\ - estrogênio \\ - vibração \\ - junção neuromuscular \\ - músculo esquelético \\ Objetivo Avaliar as junções neuromusculares (JNMs) e o tipo de fibras musculares do músculo sóleo de ratas Wistar ooforectomizadas e submetidas a um protocolo de vibração mecânica. \\ Métodos Foram utilizadas 36 ratas randomizadas nos grupos pseudo-ooforectomia sem e com tratamento e ooforectomia sem e com tratamento. O tratamento foi realizado com plataforma vibratória, frequência de $60 \mathrm{~Hz}$ e duração de 10 minutos, 3 vezes por semana, durante 4 semanas. Ao término do período de intervenção, os animais foram eutanasiados e os músculos sóleos coletados e processados para análise das JNMs e tipo de fibra. Os dados foram analisados quanto à normalidade pelo teste Shapiro-Wilk e análise da variância de 3 vias, utilizando o pós-teste de Tukey quando necessário, tendo sido adotado o nível de significância de $5 \%$. \\ Resultados $\mathrm{Na}$ análise das JNMs, o grupo ooforectomia apresentou área menor que o grupo pseudo-ooforectomia, porém o grupo ooforectomia tratado igualou-se ao grupo pseudo-ooforectomia tratado. Para o maior diâmetro das junções, o grupo ooforectomia também se apresentou diferente dos demais; porém, os animais do grupo ooforectomia tratado foram maiores que o grupo pseudo-ooforectomia tratado. Não houve distinção dos tipos de fibras, com o músculo apresentando fibras do tipo oxidativo. \\ Conclusão A privação hormonal reduziu a área e o diâmetro das JNMs, com reversão deste processo nos grupos que realizaram o tratamento com plataforma vibratória durante 4 semanas e, ainda, tanto cirurgia quanto tratamento não influenciaram no tipo de fibra do músculo sóleo, composto por fibras oxidativas.}

\footnotetext{
Trabalho feito na Universidade Estadual do Oeste do Paraná (UNIOESTE) - Campus Cascavel, PR, Brasil
}

recebido

22 de Março de 2018

aceito

10 de Julho de 2018
DOI https://doi.org/

10.1055/s-0039-1697016. ISSN 0102-3616.
Copyright $(2019$ by Sociedade Brasileira License terms de Ortopedia e Traumatologia. Published by Thieme Revinter Publicações Ltda, Rio de Janeiro, Brazil 
Abstract
Keywords
- estrogens
- vibration
- neuromuscular junction
- skeletal muscle

Objective To evaluate the neuromuscular junctions (NMJs) and the type of muscle fibers of the soleus muscle of oophorectomized Wistar rats submitted to a mechanical vibration protocol.

Methods A total of 36 randomized rats were used in the pseudo-oophorectomy without and with treatment and oophorectomy without and with treatment groups. The treatment was performed with a vibratory platform, frequency of $60 \mathrm{~Hz}$ and duration of 10 minutes, 3 times a week, for 4 weeks. At the end of the intervention period, the animals were euthanized and the soleus muscles were collected and processed for analysis of the NMJs and fiber type. The data were analyzed for normality by the Shapiro-Wilk test and analysis of the 3-way variance using the post-hoc Tukey test, when necessary, and a significance level of $5 \%$ was adopted.

Results In the analysis of the NMJs, the oophorectomy group presented a smaller area than the pseudo-oophorectomy group, but the oophorectomy with treatment group was equal to the pseudo-oophorectomy with treatment group. For the larger diameter of the joints, the oophorectomy group was also different from the others; however, the oophorectomy and treatment animals were larger than those of the pseudo-oophorectomy and treatment group. There was no distinction of the types of fibers, with the muscle presenting fibers of the oxidative type.

Conclusion Hormonal deprivation reduced the area and diameter of the NMJs, with reversion of this process in the groups that underwent vibratory platform treatment for 4 weeks, and both surgery and treatment did not influence the type of soleus muscle fiber, composed of oxidative fibers.

\section{Introdução}

A junção neuromuscular (JNM) permite a comunicação entre o sistema nervoso periférico e as fibras musculoesqueléticas, tendo como função transmitir os impulsos elétricos dos neurônios motores às miofibrilas conectadas, sendo estas responsáveis pelo desenvolvimento da força contrátil. ${ }^{1,2}$ A JNM é composta pelos compartimentos pré- e pós-sinápticos, tendo como componentes o axônio periférico, sua bainha de mielina e células de Schwann, vesículas e receptores de acetilcolina, a enzima acetilcolinesterase e a membrana basal muscular. ${ }^{3}$

Em mamíferos, as fibras musculares podem ser divididas em oxidativas (ou tipo I), oxidativas-glicolíticas (ou tipo IIa) e glicolíticas (ou tipo IIb e IIx), classificadas de acordo com a atividade metabólica. ${ }^{4}$ AsJNMs são dependentes do tipo de fibra muscular e, como no caso do músculo sóleo, as fibras oxidativas são inervadas por motoneurônios de velocidade de condução lenta e capazes de sustentar a tensão por maiores períodos. ${ }^{1}$

Uma vez que o tecido muscular é plástico, o mesmo pode sofrer modificações de características e tamanho como resposta a estímulos. ${ }^{5,6} \mathrm{O}$ estrogênio é um hormônio presente em ambos os sexos, porém mais característico no feminino. 0 sistema musculoesquelético também apresenta receptores de estrogênio, e quando as concentrações do hormônio se encontram fora da homeostase, há efeitos celulares como alteração na transcrição de proteínas, de genes e efeitos apoptóticos que irão afetar consequentemente a estrutura, o desempenho e a força muscular. ${ }^{4}$

Outro estímulo que interfere no tecido muscular é o exercício físico, capaz de promover modificações tanto no tipo de fibra quanto nas junções neuromusculares, promovendo a conversão do tipo de fibra e aumentando o número de componentes pré- e pós-sinápticos, respectivamente. ${ }^{5,6}$ Em suma, o exercício físico é utilizado como tratamento para mulheres no período pós-menopausa para melhorar a capacidade funcional, a coordenação, a força muscular e, consequentemente, a qualidade de vida. ${ }^{7,8}$ Uma modalidade de tratamento de alterações no sistema muscular é a utilização de vibrações mecânicas. Para ofertar essas vibrações, são utilizadas plataformas vibratórias que se apresentam como um método de fácil aplicabilidade e menor tempo de duração quando comparado à outras modalidades de exercício. ${ }^{9}$ No tecido muscular, essas vibrações objetivam, após alterações no comprimento músculotendíneo, o ganho de força máxima, potência e hipertrofia muscular ao exigir a contração e a manutenção da postura durante a realização do exercício, sendo um método que pode ser eficaz no tratamento de alterações decorrentes da privação hormonal no tecido muscular. ${ }^{10,11}$

No entanto, há uma escassez de dados na literatura referentes aos efeitos da privação hormonal nas junções neuromusculares e nos tipos de fibras musculares, assim como das possíveis alterações que a vibração mecânica pode causar nestas estruturas. Ainda, soma-se a busca por informações relevantes para o tratamento de desordens provenientes da privação hormonal, processo este inerente às mulheres. Desta forma, o presente estudo buscou avaliar histomorfometricamente as junções neuromusculares e o tipo de fibras do músculo sóleo de ratas Wistar ooforectomizadas e submetidas ao tratamento com vibração mecânica em plataforma comercial durante 4 semanas. 


\section{Materiais e Método}

O presente estudo teve caráter experimental, transversal e quantitativo, desenvolvido no Laboratório de Estudo de Lesões e Recursos Fisioterapêuticos e no Laboratório de Biologia Estrutural e Funcional da nossa instituição, sendo o projeto de pesquisa autorizado pelo Comitê de Ética em Uso Animal - CEUA Unioeste.

Foi realizado um delineamento inteiramente casualizado, em esquema fatorial $2 \times 2$, com o grupo amostral composto por 36 ratas fêmeas da linhagem Wistar, nulíparas, com peso médio de $177 \pm 15.8$ gramas e idade inicial de 8 semanas, obtidas do Biotério Central e mantidas no Laboratório de Estudo de Lesões e Recursos Fisioterapêuticos, em caixaspadrão de polipropileno, em ambiente com temperatura de $23 \pm 1^{\circ} \mathrm{C}$, com fotoperíodo de 12 horas, recebendo água e ração ad libitum.

Prévio ao início dos procedimentos deste trabalho, as ratas passaram pelo período de aclimatação no biotério setorial durante 1 semana. 0 processo de desenvolvimento deste estudo foi realizado a partir da realização da cirurgia de ooforectomia e pseudo-ooforectomia nos animais, seguindo com o tratamento de plataforma vibratória durante 4 semanas, findando com a eutanásia e coleta do material biológico para análises.

Os animais foram randomizados nos grupos PseudoOoforectomia (GP) e Ooforectomia (GO), submetidos à pseudocirurgia ou cirurgia efetiva de ooforectomia para retirada dos ovários. Os animais foram ainda subdivididos para grupos que passaram ou não pelo tratamento com vibração mecânica, resultando nos 4 grupos ( $n=9$ cada) do estudo: Pseudo-ooforectomia (GP); Pseudo-ooforectomia Tratamento (GPT), Ooforectomia (GO) e Ooforectomia Tratamento (GOT).

As cirurgias de pseudo-ooforectomia e ooforectomia foram realizadas em todos os animais na $8^{\mathbf{a}}$ semana de vida dos mesmos, utilizando o protocolo de Khajuria et al, $^{12}$ o mais indicado para a realização da ooforectomia em modelos experimentais por ser fácil, rápido e possibilitar uma melhor recuperação para os animais.

Para tanto, os animais foram devidamente anestesiados com injeção intraperitoneal de Dopalen $80 \mathrm{mg} / \mathrm{kg}$ e de Anasedan $20 \mathrm{mg} / \mathrm{kg}$ (Paulínea, SP, Brasil) e posteriormente à verificação do estado de consciência, observado pela ausência de resposta motora ao pinçamento da cauda e das pregas interdigitais, realizou-se a tricotomia e assepsia com álcool iodado na região de baixo ventre. Uma incisão cirúrgica longitudinal, com lâmina de bisturi número 11, foi realizada para acessar a cavidade peritoneal, sendo afastado o tecido adiposo até a identificação das tubas uterinas e ovários. Após este reconhecimento dos órgãos, foi feita uma sutura com fio catgut 4.0 simples na área dos cornos uterinos, promovendo a ressecção dos ovários bilateralmente. Ao final do procedimento, foram realizadas suturas internas com fio reabsorvível catgut 4.0 simples e as externas com fio de nylon 4.0.

A pseudo-ooforectomia consistiu na realização de todas as etapas cirúrgicas semelhantes à ooforectomia, com exceção da remoção dos ovários, para os animais passarem pelo mesmo estresse cirúrgico, evitando um viés no estudo. Subsequente à cirurgia, as ratas permaneceram 8 semanas sem qualquer intervenção, livres na gaiola para o período de indução dos efeitos da privação hormonal. ${ }^{12}$

Para o tratamento com vibração dos animais dos grupos GPT e GOT, foi utilizado um modelo de plataforma Vibro Oscilatória triplanar profissional (Arktus, Santa Tereza do Oeste, PR, Brasil). O protocolo utilizado foi adaptado de Butezloff et al, ${ }^{13}$ utilizando uma frequência de $60 \mathrm{~Hz}$ e vibrações alternadas com uma amplitude de 2 milímetros, durante 10 minutos, 3 vezes por semana. 0 tratamento foi iniciado a partir da $8^{\mathrm{a}}$ semana pós-operatória, quando os 60 dias de privação hormonal foram completados, e teve duração de 4 semanas.

Para a realização do tratamento em plataforma comercial, utilizou-se um suporte desenvolvido pelos pesquisadores de acordo com as dimensões do aparelho. $\mathrm{O}$ uso de um suporte teve como finalidade conter o animal durante $o$ tratamento com a vibração e possibilitar a realização do treinamento com vários animais de forma simultânea, otimizando-se o tempo. ${ }^{14}$ Este suporte confeccionado com madeira MDF, da cor branca, permitiu posicionar 8 animais concomitantemente em baias com 13 centímetros de largura, 19 centímetros de comprimento e altura de 25 centímetros. Ainda, para minimizar um possível viés sobre pontos distintos de aceleração e amplitude sobre a plataforma vibratória, ${ }^{15}$ foi elaborado um rodízio entre as baias, fazendo com que os animais alternassem o local em que foram alojados durante o treino ao longo dos dias.

Após o período de tratamento, os animais foram devidamente anestesiados via intraperitoneal e eutanasiados por overdose de anestésico ao final da $12^{\underline{a}}$ semana pós-operatória com ou sem plataforma vibratória. Os músculos sóleos direitos e esquerdos foram dissecados, limpos, pesados e seccionados em fragmentos com uma lâmina de inox para posterior realização das análises.

Para o estudo das JNMs, foram utilizados os fragmentos distais dos antímeros direitos, removidos e imersos no fixador Karnovisky ${ }^{16}$ em temperatura ambiente. Os músculos foram cortados longitudinalmente em quatro ou cinco porções com lâminas de inox, e os cortes obtidos foram submetidos à reação de esterase inespecífica. ${ }^{17} \mathrm{Na}$ análise morfométrica das JNMs, foram mensuradas a área e maior diâmetro de 150 JNMs por animal estudado, obtidas a partir de imagens microscópicas no aumento de 200 vezes. O fragmento distal do antímero esquerdo foi coletado e mantido em temperatura ambiente durante 40 minutos. ${ }^{18}$ Após esse período, para a preservação do tecido, o material foi coberto com talco neutro, de acordo com a técnica de Moline et $\mathrm{al}^{19}{ }^{19}$ congelado em nitrogênio líquido durante dois minutos, acondicionados em criotubos e armazenado em Biofreezer (Lupetec, São Carlos São Paulo, Brasil) a $80^{\circ} \mathrm{C}$ para análise histoenzimológica. Os segmentos musculares congelados foram transferidos para uma câmara de criostato Cryostat CM 2850 (Lupetec, Vila Monumento, SP, Brasil) a $-30^{\circ} \mathrm{C}$ e mantidos durante 30 minutos. Em seguida, os segmentos tiveram a extremidade colada a um suporte 
Tabela 1 Área e maior diâmetro dos receptores das junções neuromusculares do músculo sóleo de ratas Wistar

\begin{tabular}{|l|l|l|l|}
\hline & & Área & Maior diâmetro \\
\hline GRUPOS & GP & $5,80 \pm 0,2^{\mathrm{a}}$ & $4,25 \pm 0,1^{\mathrm{b}}$ \\
\cline { 2 - 4 } & GPT & $5,65 \pm 0,4^{\mathrm{a}}$ & $4,03 \pm 0,17^{\mathrm{b}}$ \\
\cline { 2 - 4 } & GO & $5,2 \pm 0,40^{\mathrm{b}}$ & $3,82 \pm 0,25^{\mathrm{c}}$ \\
\cline { 2 - 4 } & GOT & $5,54 \pm 0,2^{\mathrm{a}}$ & $5,54 \pm 0,13^{\mathrm{a}}$ \\
\hline
\end{tabular}

Abreviações: GO, grupo ooforectomia; GOT, grupo ooforectomia tratado; GP, grupo pseudo-ooforectomia; GPT, grupo pseudo-ooforectomia tratado. Valores expressos em média \pm desvio padrão. Letras diferentes representaram diferença estatisticamente significativa.

metálico utilizando-se um meio de congelamento de células Leica EM AFS2 (Leica Biosystems, Wetzlar, Alemanha) e foram seccionados transversalmente a $7 \mu \mathrm{m}$ de espessura. Para análise do metabolismo oxidativo e glicolítico das fibras musculares, as secções transversais foram submetidas à reação nicotinamida adenina dinucleotídeo - tetrazolium reductase (NADH-TR), conforme a técnica de Pearse ${ }^{20}$ modificada por Dubowitz et al. ${ }^{21}$ Essa análise busca quantificar e mensurar em porcentagem os diferentes tipos de fibras musculares (I, Ila e IIb) de acordo com a tonalidade apresentada nas fibras após a reação enzimática. Para cada animal, foram escolhidos aleatoriamente 3 campos microscópicos no aumento de 200 vezes.

A análise dos dados foi realizada por meio da estatística descritiva, avaliação da normalidade dos resíduos pelo teste de Shapiro-Wilk, com posterior análise da variância de três vias. Em caso de significância estatística $(p<0,05)$, foi realizado o teste Tukey-HSD, com auxílio do pacote ExpDes.pt do programa R (R Core Team, 2017).

\section{Resultados}

\section{Junções neuromusculares}

Quanto à área das JNMs, houve interação significativa entre ooforectomia e tratamento $(\mathrm{F}=4,99 ; p=0,03)$. Houve diferença entre os grupos GP e GO, demonstrando que a cirurgia de ooforectomia exerce efeitos tal como esperado, com diminuição dos valores da área das JNMs $(\mathrm{F}=15,07$; $p<0,001)$. Já o tratamento com plataforma normalizou esses valores, uma vez que os grupos GOT e GPT possuem médias equivalentes $(\mathrm{F}=0,52 ; p=0.4)$, também comprovado pela diferença entre os grupos GO e GOT $(\mathrm{F}=5,44 ; p=0,02)$.

Para o maior diâmetro das JNMs, também houve interação significativa entre ooforectomia e tratamento $(F=27,05$; $p<0,001)$. O grupo GO é diferente dos grupos GP $(\mathrm{F}=26,6$; $p<0,001)$ e GPT $(\mathrm{F}=7,06 ; p=0,01)$, significando que a privação hormonal também influencia no diâmetro das junções. Os grupos GO e GOT apresentaram diferença ( $\mathrm{F}=22,09$; $p<0.001$ ), sendo o GOT maior que o GO. Além disso, nesta variável, os valores do grupo GOT foram maiores do que os do GPT ( $F=26,6 ; p<0,001)$, reforçando um possível efeito da plataforma vibratória no diâmetro ( - Tabela 1). Essas alterações morfométricas também são visualizadas na - Fig. 1, representando a morfologia dessas JNMs.

A -Fig. 2 ilustra as JNMs nos grupos utilizados no presente trabalho, com as mesmas possuindo formas elípticas e ovais. Morfologicamente, é possível verificar a diminuição da área e, consequentemente, menor diâmetro das JNMs do grupo GO (-Fig. 1C), comprovado também pela morfometria (-Tabela $\mathbf{1}$ )

\section{Tipo de fibras musculares}

Analisando os músculos sóleos após a técnica NADH-TR (-Fig. 2), foi evidenciado que não houve diferenciação das
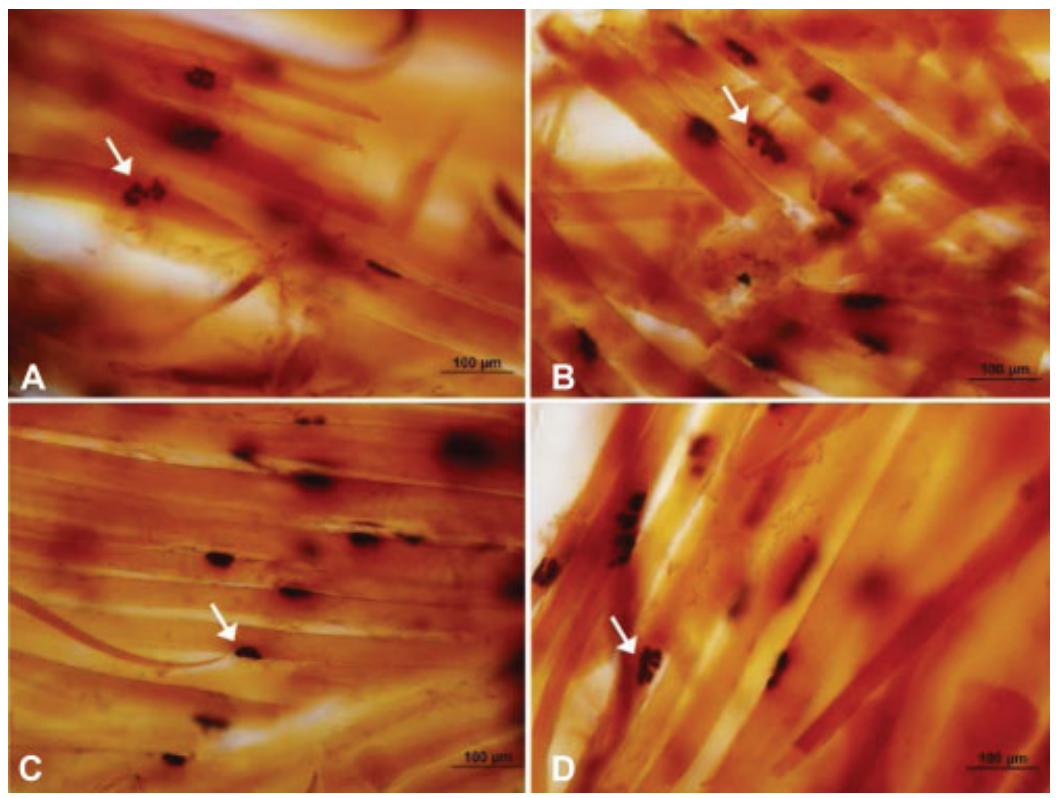

Fig. 1 Fotomicrografias dos receptores de junções neuromusculares do músculo sóleo de ratas Wistar após reação de esterase inespecífica. Em A, grupo pseudo-ooforectomia (GP); B, grupo pseudo-ooforectomia tratado (GPT); C, grupo ooforectomia (GO) com diminuição da área e diâmetro das junções neuromusculares quando comparado morfologicamente aos demais grupos; D, grupo ooforectomia tratado (GOT). Ponta de seta representa os receptores das junções neuromusculares marcados com a técnica. 


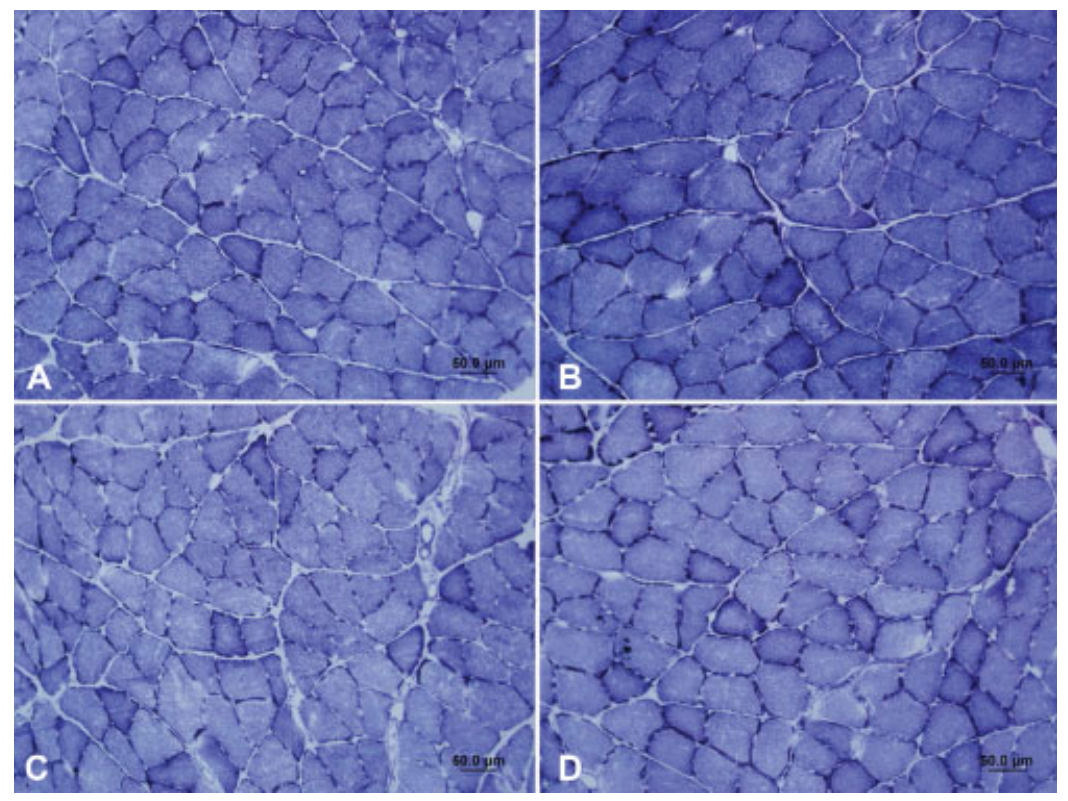

Fig. 2 Fotomicrografias do músculo sóleo de ratas Wistar, corte transversal, reação enzimática nicotinamida adenina dinucleotídeo tetrazolium reductase. Em A, Grupo pseudo-ooforectomia (GP); B Grupo pseudo-ooforectomia tratado (GPT); C grupo ooforectomia (GO) e D, grupo ooforectomia tratado (GOT). Não houve diferenciação entre a coloração das fibras musculares nesta técnica, com as fibras apresentandose em tua totalidade como fibras do tipo I.

fibras musculares oxidativas (I), intermediárias (IIa) e glicolíticas (IIb), uma vez que não houve alternância entre a intensidade das colorações características da técnica.

\section{Discussão}

Como resultados, foi possível observar que a cirurgia de ooforectomia e o tratamento com a plataforma vibratória foram capazes de influenciar apenas a morfometria e morfologia das JNMs, não afetando o tipo de fibra muscular do sóleo das ratas. Em relação à análise das JNMs, foi possível observar que o efeito da privação hormonal obtido pela cirurgia de ooforectomia exerceu influência sobre estas estruturas, com diminuição dos valores tanto de área quanto de diâmetro. Embora não haja estudos que avaliem especificamente as JNMs na privação hormonal, a presença de receptores sensíveis ao estrogênio nos tecidos relacionados ${ }^{4}$ pode justificar alterações observadas nesse modelo experimental.

Ainda não há consenso sobre efeitos patológicos do estrogênio no tecido nervoso, e existe uma hipótese de que ele possa exercer influência, atuando no processamento sensorial e na modulação da transmissão nociceptiva. ${ }^{22}$ Contudo, não se pode afirmar que as alterações encontradas nas JNMs no presente trabalho sejam decorrentes do tecido nervoso, uma vez que as mesmas podem ser afetadas fisiopatologicamente tanto por entrada pré quanto pós-sináptica. ${ }^{23-25}$ Desta forma, a própria degeneração da JNM pode ter sido desencadeada pela perturbação no metabolismo muscular seguida de alterações nas unidades motoras. ${ }^{26,27}$

$O$ déficit de estrogênio, causado experimentalmente pela cirurgia de ooforectomia, pode estar relacionado com algumas perturbações no metabolismo muscular que culminam no aumento de marcadores inflamatórios e na alteração no metabolismo mitocondrial, ${ }^{28}$ podendo levar a uma sensibilização e à perda de unidades motoras, diminuição da massa e atrofia muscular, ${ }^{29}$ pela degeneração das JNMs, ${ }^{26,27}$ corroborando com os achados desse estudo.

Deschenes et $\mathrm{al}^{2}$ investigaram os efeitos do gênero e da diminuição da descarga de peso após 2 semanas de elevação do membro posterior. Como resultado, concluíram que as JNMs não diferiram entre os sexos masculino e feminino nos grupos controle, com condições fisiológicas padrão, e o período de 2 semanas de intervenção não influenciou a morfometria e a morfologia das JNMs nos músculos sóleo, extensor longo dos dedos e plantar, sendo a transmissão sináptica equivalente nos dois sexos em condições normais. De forma contrária, em nosso estudo, as JNMs foram afetadas no grupo que sofreu privação hormonal. Assim, é possível assumir que as alterações sejam dependentes do tempo em que o estímulo é mantido, uma vez que em um trabalho anterior do mesmo grupo de pesquisa, Deschenes et al ${ }^{30}$ verificaram que 4 semanas de diminuição da descarga foi capaz de ocasionar mudanças nas JNMs.

Também não existem dados na literatura do comportamento das JNMs frente à vibração mecânica. Entretanto, com o presente estudo, foi possível verificar que o tratamento com a plataforma vibratória foi eficaz na reversão dos efeitos deletérios da privação hormonal, uma vez que o grupo GOT se apresentou semelhante aos grupos pseudo-ooforectomia na variável área e até maior que os mesmos em relação ao diâmetro. Tanto a diminuição quanto o aumento da atividade física são capazes de ocasionar mudanças nas JNMs, influenciando a área e o comprimento dos ramos e, consequentemente, a relação pré- e pós-sináptica, havendo uma hipertrofia das JNMs compensatória ao exercício. ${ }^{1} 0$ treinamento de resistência realizado por Deschenes et al, ${ }^{25}$ com 6 semanas de esteira, foi capaz de promover a remodelação das JNMs do 
músculo plantar, mas não do extensor longo dos dedos, ou seja, houve efeitos no músculo mais recrutado durante o exercício executado, sugerindo que os resultados também são dependentes do recrutamento muscular utilizado.

De acordo com Nishimune et $\mathrm{al}^{23}{ }^{23}$ o músculo sóleo é muito utilizado em estudos para verificar as adaptações das JNMs perante o exercício físico devido à homogeneidade na composição do tipo de fibra muscular e a função antigravitacionária, com os resultados dependentes do tipo, intensidade e finalidade do exercício com o tipo de músculo. Seene et a ${ }^{1}$ buscaram avaliar as diferenças entre as JNMs de fibras oxidativas e glicolíticas, bem como o comportamento das mesmas durante o exercício físico. Nas fibras glicolíticas, os terminais e fibras axônicas são elípticas e maiores que os de fibras lentas, ocupando uma maior área no sarcoplasma. Já nas fibras oxidativas, mais presentes no músculo sóleo, os terminais axônicos são curtos, redondos e morfologicamente semelhantes, com também maior número de mitocôndrias. Os dois tipos de fibras são afetados com o treinamento; porém, neste estudo de seis semanas de caminhada em esteira, houve maior remodelação das JNMs nas fibras oxidativas, provavelmente devido à modalidade do exercício escolhido exigir mais destas fibras. Ainda, esses mesmos autores ${ }^{1,23}$ também utilizaram as técnicas de imunofluorescência e citofluorescência e verificaram que o exercício físico proporcionou acréscimo no número de vesículas nos terminais axônicos, isto atribuído à ressíntese de acetilcolina após a sinapse, proporcionando a remodelação das junções. Apesar da modalidade de exercício ter sido outra, os efeitos benéficos do tratamento com a plataforma vibratória também podem se basear nessa hipótese da literatura; entretanto, são necessárias metodologias mais específicas para a confirmação.

De acordo com Haizlip et al, ${ }^{4}$ há uma prevalência de fibras oxidativas no sexo feminino quando comparado ao masculino. Esta característica promove maior desempenho, resistência e melhor recuperação em resposta ao exercício e fadiga muscular. Embora apenas fêmeas tenham sido avaliadas em nosso estudo, a informação citada reforça os resultados do tratamento. Ainda que o presente artigo tenha sido realizado com modalidades diferentes de exercício, o protocolo de exercício com a vibração mecânica utilizado exigia a manutenção da postura dos animais durante exposição à vibração mecânica e, consequentemente, este padrão de recrutamento pode explicar os resultados positivos encontrados.

Diante do exposto, é visto que o exercício físico possui demasiada importância na manutenção da funcionalidade física das JNMs. Outra possível explicação da adaptação das JNMs frente ao tratamento com a plataforma vibratória pode ser pelo fato da contração muscular exigida pelo exercício interferir na regulação e expressão proteica de moléculas e fatores de crescimento como o fator neurotrófico derivado de células da glia (GDNF, na sigla em inglês), fator de crescimento semelhante à insulina 1 (IGF-1, na sigla em inglês) e interleucinas como a IL1-Ra, IL-10 e até mesmo a IL-6, levando à adaptação, à melhora da transmissão e à hipertrofia das JNMs. ${ }^{3,23,31}$

Em relação ao tipo de fibra, o músculo sóleo é composto principalmente por fibras do tipo I em todos os grupos, tendo esse tipo de fibra maior capacidade de respiração mitocondrial, ${ }^{3}$ com a técnica de NADH-TR sendo utilizada para determinar o funcionamento oxidativo das mitocôndrias por meio da coloração após a reação enzimática. ${ }^{32}$ A cirurgia de ooforectomia também não afetou a porcentagens de cada tipo de fibra no músculo sóleo de camundongos no trabalho de Moran et $\mathrm{al}^{33}$ porém com análise imunohistoquímica foram encontrados os diferentes tipos de fibras. Haizlip et $\mathrm{al}^{4}$ também concluíram que em modelos de ooforectomia não houve alterações no tipo de fibra e somente mudanças no tamanho e diâmetro muscular. Embora em menor número, esta diferenciação poderia ter sido encontrada utilizando técnicas mais específicas como, por exemplo, imunofluorescência e atividade da ATPase.

Assim como a cirurgia de ooforectomia, o tratamento com a plataforma vibratória não alterou o tipo de fibra muscular com a análise utilizada, embora a literatura aponte que o exercício físico também é capaz de promover alterações no tipo de fibra. ${ }^{1}$ Camargo Filho et $\mathrm{al}^{34}$ analisaram a resposta do músculo sóleo frente ao exercício de esteira e exposição ao cigarro. Foram observados diferentes níveis de atividade enzimática das fibras; entretanto, assim como neste estudo, a reação de NADH-TH identificou em sua maioria fibras oxidativas. No trabalho de Deschenes et $\mathrm{al}^{2}{ }^{2}$ avaliando também o músculo sóleo, não houve mudanças no tipo de fibra, uma vez que $>95 \%$ das fibras musculares deste músculo, para ambos os sexos, era composta por fibras do tipo I. A pequena porcentagem de fibras do tipo II do sóleo não apresentou efeitos significativos para o sexo e tratamento. Músculos posturais profundos podem expressar um maior número de fibras com fenótipo oxidativo (fibras I e Ila), diferentemente de músculos mais superficiais como o gastrocnêmio (fibras IIx e IIb). ${ }^{4}$ No presente trabalho, utilizou-se o músculo sóleo, e atribuise às características dele ser o principal músculo postural e oxidativo no rato ${ }^{3}$ o fato de não terem sido encontrados diferentes tipos de fibra.

\section{Conclusão}

Com base nos resultados do presente estudo, foi possível concluir que a área e o diâmetro das JNMs foram afetados tanto pela cirurgia de ooforectomia quanto pelo tratamento com a plataforma vibratória, sendo este capaz de reverter alterações morfométricas e morfológicas. Ainda, conclui-se que o músculo sóleo de ratas Wistar é predominantemente composto por fibras oxidativas que não foram alteradas com o modelo experimental de privação hormonal e tratamento com vibração mecânica.

Conflitos de Interesses

Os autores declaram não haver conflitos de interesses.

Agradecimentos

À Fundação Araucária pelos recursos financeiros (edital 09/2016 - Pesquisa Básica e Aplicada) e ao Conselho Nacional de Desenvolvimento Científico e Tecnológico (CNPq) pela concessão de bolsa para auxílio da pesquisa. 


\section{Referências}

1 Seene T, Umnova M, Kaasik P. Morphological peculiarities of neuromuscular junctions among different fiber types: Effect of exercise. Eur J Transl Myol 2017;27(03):6708

2 Deschenes MR, Adan MA, Kapral MC, et al. Neuromuscular adaptability of male and female rats to muscle unloading. J Neurosci Res 2018;96(02):284-296

3 Krause Neto W, Ciena AP, Anaruma CA, de Souza RR, Gama EF. Effects of exercise on neuromuscular junction components across age: systematic review of animal experimental studies. BMC Res Notes 2015;8:713

4 Haizlip KM, Harrison BC, Leinwand LA. Sex-based differences in skeletal muscle kinetics and fiber-type composition. Physiology (Bethesda) 2015;30(01):30-39

5 Baehr LM, West DW, Marcotte G, et al. Age-related deficits in skeletal muscle recovery following disuse are associated with neuromuscular junction instability and ER stress, not impaired protein synthesis. Aging (Albany NY) 2016;8(01):127-146

6 Pour MB, Joukar S, Hovanloo F, Najafipour H. Long-term lowintensity endurance exercise along with blood-flow restriction improves muscle mass and neuromuscular junction compartments in old rats. Iran J Med Sci 2017;42(06):569-576

7 Radominski SC, Bernardo W, Paulac AP, et al. Diretrizes brasileiras para o diagnóstico e tratamento da osteoporose em mulheres na pós-menopausa. Rev Bras Reumatol 2017;57(S2):S452-S66

8 Caputo EL, Costa MZ. Influência do exercício físico na qualidade de vida de mulheres pós-menopáusicas com osteoporose. Rev Bras Reumatol 2014;54(06):467-473

9 Anwer S, Alghadir A, Zafar H, Al-Eisa E. Effect of whole body vibration training on quadriceps muscle strength in individuals with knee osteoarthritis: a systematic review and meta-analysis. Physiotherapy 2016;102(02):145-151

10 Park SY, Son WM, Kwon OS. Effects of whole body vibration training on body composition, skeletal muscle strength, and cardiovascular health. JExerc Rehabil 2015;11(06):289-295

11 Cerciello S, Rossi S, Visonà E, Corona K, Oliva F. Clinical applications of vibration therapy in orthopaedic practice. Muscles Ligaments Tendons J 2016;6(01):147-156

12 Khajuria DK, Razdan R, Mahapatra DR. Descrição de um novo método de ooforectomia em ratas. Rev Bras Reumatol 2012;52 (03):466-470

13 Butezloff MM, Zamarioli A, Leoni GB, Sousa-Neto MD, Volpon JB. Whole-body vibration improves fracture healing and bone quality in rats with ovariectomy-induced osteoporosis. Acta Cir Bras 2015;30(11):727-735

14 Brouwers JE, van Rietbergen B, Ito K, Huiskes R. Effects of vibration treatment on tibial bone of ovariectomized rats analyzed by in vivo micro-CT. J Orthop Res 2010;28(01):62-69

15 Alizadeh-Meghrazi M, Zariffa J, Masani K, Popovic MR, Craven BC. Variability of vibrations produced by commercial whole-body vibration platforms. J Rehabil Med 2014;46(09):937-940

16 Karnovsky MJ. A formaldehyde-glutaraldehyde fixative of high osmolality for use in electron microscopy. JCell Biol 1965;27:1A-149A
17 Lehrer GM, Ornstein L. A diazo coupling method for the electron microscopic localization of cholinesterase. JBiophys Biochem Cytol 1959;6:399-406

18 Khan MA. The histoenzymology of striated muscle fibres: an overview. Cell Mol Biol Incl Cyto Enzymol 1977;22(3-4):383-393

19 Moline SW, Glenner GG. Ultrarapid tissue freezing in liquid nitrogen. J Histochem Cytochem 1964;12:777-783

20 Pearse AG. Histochemistry: theoretical and applied. 3rd ed. Baltimore: Williams \& Wilkins; 1972

21 Dubowitz V, Brooke M. Muscle biopsy: a mordern approach. London: Saunders; 1973

22 Amandusson $\AA$, Blomqvist A. Estrogenic influences in pain processing. Front Neuroendocrinol 2013;34(04):329-349

23 Nishimune H, Stanford JA, Mori Y. Role of exercise in maintaining the integrity of the neuromuscular junction. Muscle Nerve 2014; 49(03):315-324

24 Rudolf R, Khan MM, Labeit S, Deschenes MR. Degeneration of neuromuscular junction in age and dystrophy. Front Aging Neurosci 2014;6:99

25 Deschenes MR, Kressin KA, Garratt RN, Leathrum CM, Shaffrey EC. Effects of exercise training on neuromuscular junction morphology and pre- to post-synaptic coupling in young and aged rats. Neuroscience 2016;316:167-177

26 Messier V, Rabasa-Lhoret R, Barbat-Artigas S, Elisha B, Karelis AD, Aubertin-Leheudre M. Menopause and sarcopenia: A potential role for sex hormones. Maturitas 2011;68(04):331-336

27 Sipilä S, Finni T, Kovanen V. Estrogen influences on neuromuscular function in postmenopausal women. Calcif Tissue Int 2015;96 (03):222-233

28 Gonzalez-Freire M, de Cabo R, Studenski SA, Ferrucci L. The neuromuscular junction: aging at the crossroad between nerves and muscle. Front Aging Neurosci 2014;6:208

29 Carson JA, Manolagas SC. Effects of sex steroids on bones and muscles: Similarities, parallels, and putative interactions in health and disease. Bone 2015;80(01):67-78

30 Deschenes MR, Wilson MH. Age-related differences in synaptic plasticity following muscle unloading. J Neurobiol 2003;57(03): 246-256

31 Terra R, Silva SA, Pinto VS, Sutra PML. Effect of exercise on the immune system: response, adaptation and cell signaling. Rev Bras Med Esporte 2012;18(03):208-214

32 Figueiredo Braggion G, Ornelas E, Carmona Sattin Cury J, et al. Morphological and biochemical effects on the skeletal muscle ofovariectomized old female rats submitted to the intake of diets with vegetable or animal protein and resistance training. Oxid Med Cell Longev 2016;2016:9251064

33 Moran AL, Nelson SA, Landisch RM, Warren GL, Lowe DA. Estradiol replacement reverses ovariectomy-induced muscle contractile and myosin dysfunction in mature female mice. JAppl Physiol (1985) 2007;102(04):1387-93

34 Camargo Filho JC, Garcia BC, Kodama FY, et al. Efeitos do exercício aeróbio no músculo esquelético de ratos expostos à fumaça de cigarro. Rev Bras Med Esporte 2011;17(06):416-419 